\title{
Renal Transplantation in Diabetic Patients with or Without Simultaneous Pancreatic Transplantation 1986: Data from the EDTA Registry
}

\author{
G. Tufveson \\ H. Brynger \\ E. Dimeny
}

F. P. Brunner*

Transplantation Unit, University

Hospital, Uppsala, Sweden

Department of Surgery 1,

Sahlgrenska, Göteborg, Sweden

Department of Nephrology,

University Hospital, Uppsala, Sweden

J. H. H. Ehrich

Departement für Innere Medizin,

Universität Basel, Switzerland

${ }^{*}$ Chairman

\author{
W. Fassbinder \\ W. Geerlings \\ G. Rizzoni
}

N. H. Selwood

A. J. Wing
Städtische Kliniken Fulda, Federal Republic of Germany

Stichting Thuisdialyse Noord

Nederland, Haren, The Netherlands

Ospedale Pediatrico Bambino Gesú, Istituto di Ricerca Scientifica,

Roma, Italy

UK Transplant Service, Bristol, United Kingdom

St Thomas' Hospital, London, United Kingdom

\begin{abstract}
This report summarises the outcome of 90 combined kidney/pancreatic grafts performed in Europe in 1986. Data for the combined kidney/pancreas grafts were obtained by a special questionnaire. The one-year patient and kidney graft survival is compared to the results of a group of 389 patients with diabetic nephropathy on the EDTA Registry data file who received kidney grafts alone. The recipients of combined kidney-pancreas grafts were younger, whereas a greater proportion of males received kidney graft alone. Patient survival at one year after transplantation was similar: $89 \%$ in recipients of combined transplants compared to $90 \%$ in recipients of kidney grafts alone. Kidney graft survival was $78 \%$ at one year for recipients of combined grafts versus $76 \%$. It is concluded that pancreas transplantation has little effect on the fate of concomitant kidney grafts. The procedure should - in
\end{abstract}

Correspondence and offprint requests to: Professor F. P. Brunner, EDTA Registry, St Thomas' Hospital, London, SE1 7EH, UK. experienced hands and in selected patients - be almost as safe as kidney grafting alone.

Key words: Diabetic nephropathy; Combined kidney/ pancreas transplantation

\section{Introduction}

Ever since the advent of combined pancreas and kidney grafting in patients with diabetic nephropathy, the advantages and hazards of pancreatic grafting have been a matter of controversy. The impact of pancreatic grafting on patient survival and on the fate of the simultaneously transplanted kidney graft have been discussed extensively [1-4]. In 1984 McMaster and coworkers [4] suggested that combined grafting was not hazardous either to the patient or to the kidney graft. However, this conclusion was based on a small number 
of cases. Data presented from the International Pancreas Registry in Minnesota [5] showed reasonably good kidney graft survival, even though an adequate control group of patients receiving kidney grafts without pancreatic grafts was lacking.

To analyse further the question of patient and graft survival, the EDTA Registry investigated the results of 90 combined kidney/pancreas grafts performed in 1986 in Europe.

\section{Material and Methods}

A total of 122 combined kidney/pancreas transplants performed in 1986 in Europe were brought to the attention of the EDTA Registry through the 1986 centre questionnaire supplemented by information from National Keymen and other sources. A total of 96 special questionnaires were sent in early 1988 to all centres that reported having performed two or more combined grafts in 1986. Information was sought on individual graft recipients concerning sex, date of birth, date of transplantation, date of failure of pancreas/ or kidney graft, date of death, or whether the patient was alive at the end of 1987 . Of the 96 questionnaires, 90 (93.8\%) were completed and returned.

A control group was selected from the EDTA patient file. This control group consisted of all (389) reported kidney grafts in patients with diabetic nephropathy performed in 1986 in the following countries: Belgium, Denmark, Federal Republic of Germany, Finland, France, Switzerland, and the United Kingdom. Care was taken to exclude patients from these countries who had been subjected to combined kidney and pancreas grafting. Actuarial patient and graft survival rates were computed when the complete patient data up to the end of 1987 were available.

In all instances, standard actuarial methodology was used. Patient death with a functioning graft was counted as graft failure in the calculations.

\section{Results}

Table 1 depicts the distribution of the combined kidney/ pancreas grafts in 1986 by country. There was a relative predominance of combined grafts in Sweden and Norway. The remainder of the grafts were performed in Central Europe and relatively few in France, the United Kindgom, and Italy. Because of the uneven distribution of the combined grafts if was felt that a control group of recipients of kidney grafts alone should be restricted to a similar geographical area. However, Sweden and Norway were omitted as it was felt that the remainder of the diabetic graft recipients in these Scandinavian countries who had not received combined grafts might introduce a bias through a selection of higher-risk patients.

Table 1. Number of combined kidney/pancreatic transplants performed in 1986 according to country. In 1986, 26 centres reported performing 122 combined kidney/pancreatic transplants

\begin{tabular}{lc}
\hline Country & Transplants $(n)$ \\
\hline Austria & 5 \\
Belgium & 3 \\
Czechoslovakia & 4 \\
France & 13 \\
FRG & 25 \\
GDR & 2 \\
Italy & 3 \\
Netherlands & 10 \\
Norway & 10 \\
Spain & 4 \\
Sweden & 31 \\
Switzerland & 4 \\
UK & 8 \\
Total performed in 1986 & 122 \\
\hline
\end{tabular}

\section{Age and Sex Distribution}

Figure 1 shows the age and sex distribution of patients who underwent combined kidney and pancreas grafting (Fig. 1a) and those who received a kidney graft alone (Fig. 1b). In general, recipients of combined grafts tended to be younger. The median age at transplantation was 36 years for patients with combined grafts and, at 39 years, was slightly greater for recipients of kidney grafts alone (non-significant). Only three patients who received combined grafts were between 50 and 60 years old, whereas $16 \%$ of the kidney recipients were aged between 50 and 60 years, and $3 \%$ were over 60 years old. The sex distribution of $53 \%$ males versus $47 \%$ females for recipients of combined grafts differed strikingly from the patients receiving kidneys alone, of whom $65 \%$ were male and $35 \%$ female.

\section{Patient and Graft Survival}

The patient survival is shown in Figs $2 a$ and $2 b$. It was almost equal in the two groups, with $89 \%$ survival at 1 year for recipients of combined grafts compared to $90 \%$ for recipients of kidney graft alone. The kidney graft survival also differed little, with a 1-year survival of $78 \%$ with combined grafting versus $76 \%$ in patients without pancreatic grafts. The pancreas graft survival shown in Fig. 2a was $65 \%$ at 1 year. 


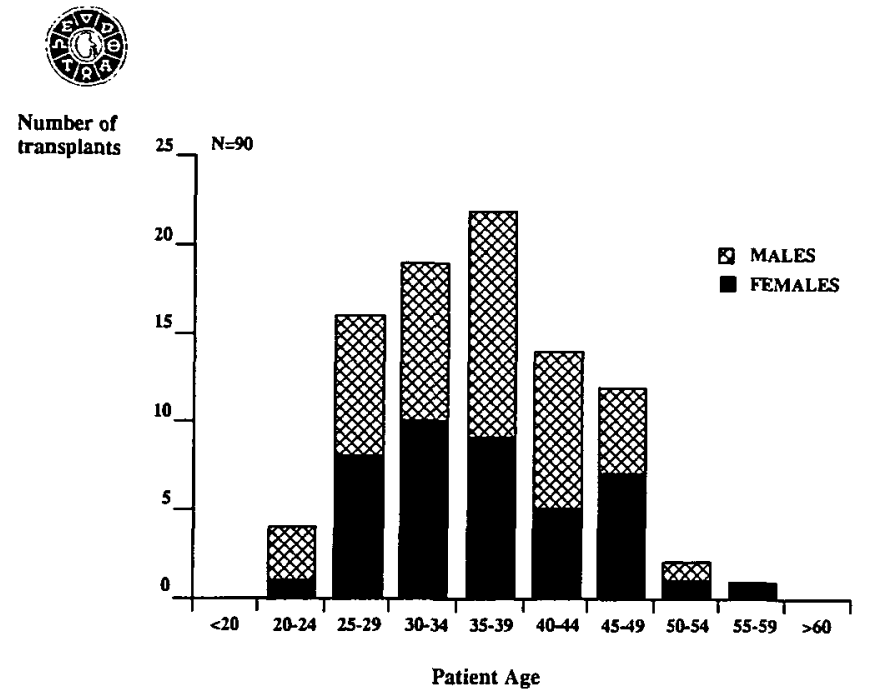

Fig. 1a. Age distribution at combined kidney and pancreas transplantation for diabetic ESRF, 1986.

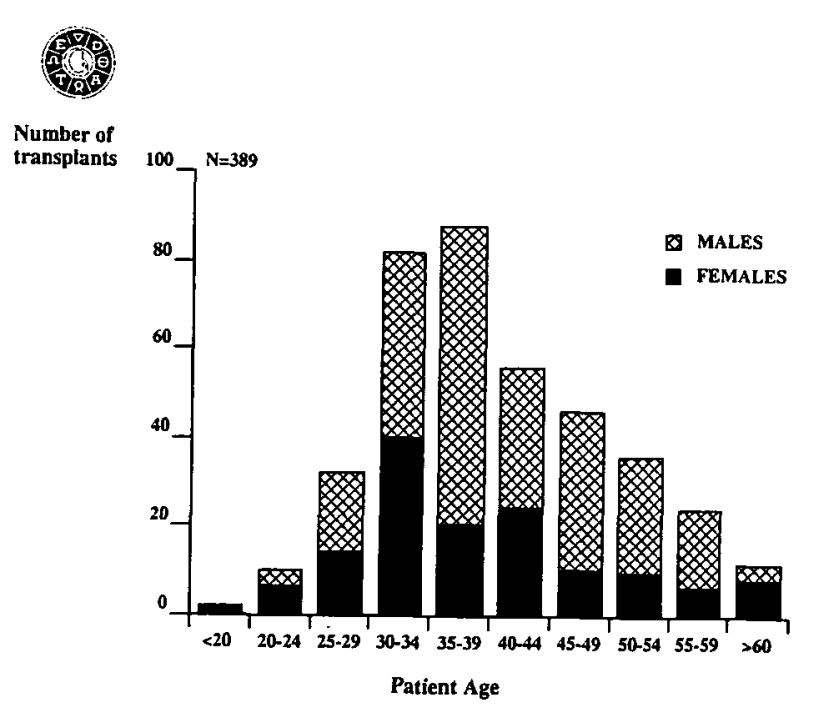

Fig. 1b. Age distribution at kidney transplantation for diabetic ESRF, 1986.

\section{Discussion}

The present report demonstrates that both patient and kidney graft survival are comparable in patients undergoing either kidney transplantation alone or combined with pancreas transplantation. The outcome of kidney transplantation with concomitant pancreas grafting was compared to the results of kidney grafting in patients with diabetic nephropathy who underwent transplantation during the same year and who were from a similar geographical area. Although this comparison cannot match the criteria of a randomised study, it nevertheless provides a useful comparison of the two surgical approaches. Details of sex and age distribution suggest
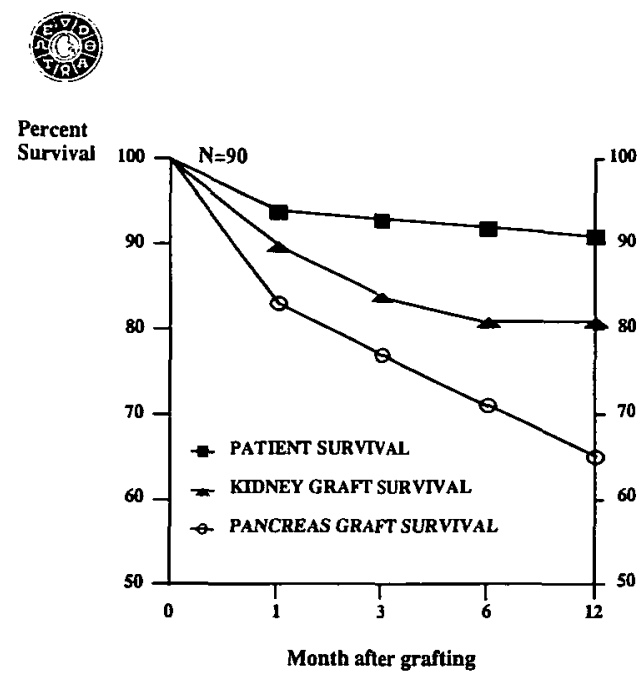

Fig. 2a. Diabetic ESRF: Survival after combined kidney and pancreas transplantation, 1986.

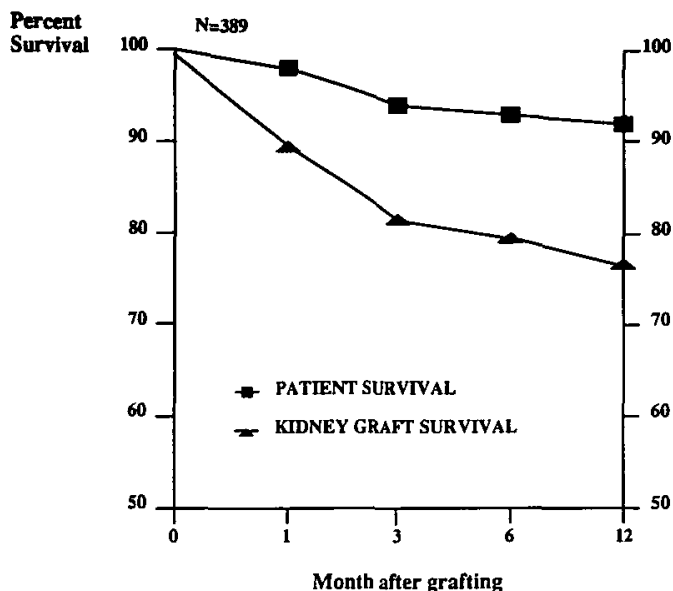

Fig. 2b. Diabetic ESRF: Survival after kidney transplantation, 1986.

that the two groups are not entirely comparable. For unknown reasons, females appeared to be accepted for combined transplantation at the slightly higher proportion of $47 \%$ as compared to the $42 \%$ female prevalence with diabetic nephropathy $(44 \%$ for type I diabetes mellitus) among patients accepted for renal replacement therapy in 1983-1985 [6]. Similarly, and in contrast to recipients of combined grafts, male patients with diabetic nephropathy were selected preferentially for kidney transplantation alone, as shown by the sex ratio of $66 \%$ males versus $34 \%$ females. These sex imbalances between the two groups should, in any event, have little influence on patient and graft survival. The results of kidney transplantation have long been 
known to be identical or very similar [7] when comparing males with females. The difference in age distribution (which is not statistically significant) would be predicted to result in slightly better patient survival in the younger recipients of combined grafts, whilst the effect on kidney graft survival is likely to be small [8]. That no such differences were apparent might be due to the relatively small number of patients included in the present study. Also, the much improved patient survival rates attained in recent years makes detection of small differences very difficult.

As the early postoperative hazards following combined kidney and pancreas grafting now appear to be comparable to those of kidney grafting alone, and the kidney graft survival is not adversely affected by pancreatic grafting, it seems justified to continue to perform combined grafts, at least in surgical units with the appropriate experience [9]. The outcome of pancreatic function in patients undergoing combined grafting is also encouraging, since only $20 \%$ of the patients with a functioning kidney graft had lost their simultaneously transplanted pancreas within the first year. Most of the pancreatic graft losses were confined to the first three months after transplantation, and the survival of a pancreas graft alone after kidney graft failure seems to be a rare event (one patient only in this series).

It is the intention of the EDTA Registry to obtain follow-up information on the present cohort of patients with combined kidney/pancreas grafts within 2-3 years, in order to establish any possible beneficial long-term effects of simultaneous pancreas grafting.
Acknowledgements. The work of the EDTA Registry has been supported by grants from Governments of National Societies of Nephrology. Grants have also been made by the AKZO Group, Asahi Medical Co. Ltd, B. Braun Melsungen AG, Baxter Healthcare Corporation, Bellco SpA, CD Medical International Ltd, Cilag AG Research, Cobe International, Fresenius AG, Gambro AB, Hospal Ltd, and Sandoz AG.

We thank all those doctors and their staff who have kindly completed EDTA Registry questionnaires, and particularly those who completed the special questionnaire on combined kidney/pancreas grafts that made this study possible.

\section{References}

1. Bohman S-O, Tydén G, Wilczek H et al. Prevention of kidney graft diabetic nephropathy by pancreas transplantation in man. Diabetes 1985; 34: 306-308

2. Pyke D. Pancreas transplantation for diabetes? Lancet 1988; 1 : 816-817

3. Sutherland DER. Who should get a pancreas transplant? Diabetes Care 1988; 11: 681-685

4. McMaster P, Michael J, Adu D et al. Combined kidney and pancreas grafting - is it really safe and does it jeopardise the kidney? Transplant Proc 1984; 16: 704-706

5. Sutherland DER, Moudry KC, Fryd DS. Results of pancreas transplant registry. Diabetes 1989; 38 [Suppl 1]: 46-54

6. Brunner FP, Brynger $\mathrm{H}$, Challah $\mathrm{S}$ et al. Renal replacement therapy in patients with diabetic nephropathy, 1980-1985. Nephrol Dial Transplant 1988; 3: 585-595

7. Brunner FP, Wing AJ, Dykes SR et al. International review of renal replacement therapy: Strategies and results. In: Maher JF, ed. Replacement of Renal Function by Dialysis. Kluwer Academic Publishers, Dordrecht, Holland, 1989; 697-719

8. Brunner FP, Broyer $M$, Brynger $\mathbf{H}$ et al. Survival on renal replacement therapy: Data from the EDTA Registry. Nephrol Dial Transplant 1988; 3: 109-122

9. Groth CG. Pancreas transplantations. Lancet 1988; 1: 1400

Received for publication 16.5.90

Accepted in revised form 10.10 .90 